\title{
Enhancing optical isolator performance in nonreciprocal waveguide arrays
}

\author{
Miguel Levy, ${ }^{1,2, *}$ Turhan K. Carroll, $^{2}$ and Ramy El-Ganainy ${ }^{1}$ \\ ${ }^{1}$ Department of Physics, Michigan Technological University, Houghton, Michigan 49931, USA \\ ${ }^{2}$ Air Force Research Laboratory, Sensors Directorate, Wright-Patterson AFB, Ohio 45433, USA \\ *Corresponding author: mlevy@mtu.edu
}

Received October 2, 2014; revised November 17, 2014; accepted November 18, 2014; posted November 20, 2014 (Doc. ID 224377); published December 24, 2014

\begin{abstract}
We investigate the operation of optical isolators based on magneto-optics waveguide arrays beyond the coupled mode analysis. Semi-vectorial beam propagation simulations demonstrate that evanescent tail coupling and the effects of radiation are responsible for degrading the device's performance. Our analysis suggests that these effects can be mitigated when the array size is scaled up. In addition, we propose the use of radiation blockers in order to offset some of these effects, and we show that they provide a dramatic improvement in performance. Finally, we also study the robustness of the system with respect to fabrication tolerances using the coupled mode theory. We show that small, random variations in the system's parameters tend to average out as the number of optical guiding channels increases. (c) 2014 Optical Society of America

OCIS codes: (130.2790) Guided waves; (130.3120) Integrated optics devices; (230.3240) Isolators; (230.7370) Waveguides.

http://dx.doi.org/10.1364/OL.40.000111
\end{abstract}

Optical isolators have been investigated extensively over the last few decades. Driven by industrial demands, much effort has been dedicated to the miniaturization and integration of these optical components in photonic circuits [1-10]. Along these lines, various prototypes with varying degrees of isolation efficiency (the ratio of backreflected light reaching the source to forward transmitted light) and forward transmission efficiency have been proposed and implemented. Despite these intense investigations, current on-chip optical isolators do not yet meet commercial standards. In order to achieve this goal, optical isolation and forward transmission performance should be further enhanced. In addition, robustness and device tolerance with respect to fabrication errors must be also addressed.

Recently, a new paradigm for designing optical isolators based on waveguide arrays was proposed $[11,12]$. In that work, a nonuniform waveguide array in which the coupling coefficients follow a square root pattern is used to transfer an incident optical signal from one end of the array to the other in the forward propagation direction. This process is known as coherent optical transport [12]. By introducing a nonreciprocal linear shift in the backward propagation direction, any reflected power from the output channel will undergo surface Bloch oscillations and will be completely redirected away from the input waveguide [see Fig. 1(b)]. The nonreciprocal effects in the latter case can be induced by depositing magnetic garnet films on top of the waveguide array [5-8]. In [12], this system was analyzed using the coupled mode theory (CMT). Despite its adequacy, this approach neglects some effects. For instance, it does not account for the reduction in isolation ratio due to the abrupt transition between the array and free space at the output. It also neglects the effects of mode conversion from an input fiber or a single-waveguide into a superposition of normal modes in the array. Thus, it shows that perfect isolation is, in principle, possible. In reality, this sharp transition, together with the evanescent field coupling between adjacent waveguides and the effects of mode conversion, will degrade the isolation coefficient. We also note that, due to the sharp discontinuity between the array and the adjacent optical stages at both input and output ends, a small part of the optical power will couple to radiation modes, thus reducing the forward transmission. Similar dynamics in the backward propagation direction will result in some light coupling to the input channel, and hence reduce the isolation efficiency. In addition, optical power leakage to radiation modes at refractive index discontinuities can also degrade the performance.

In this work, we employ an accurate unidirectional beam propagation technique to investigate these processes in more detail. We show that the effects of evanescent tail coupling can be reduced by increasing the number of waveguide elements. Furthermore, in situations where smaller device footprints are desirable, we utilize optical absorbers, which can prevent the coupling between radiation modes and waveguide channels. Our numerical analysis indicates that these strategies enhance the performance of these structures. Finally, we also study the robustness of these optical isolators with respect to fabrication errors by using the CMT when random variations in the design parameters (coupling coefficients and propagation constants) are introduced. Here

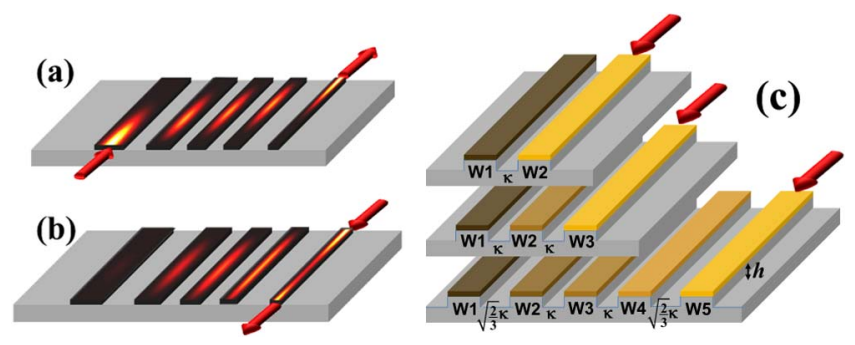

Fig. 1. Operation of the device for a five-channel array (a) forward and (b) backward. (c) Arrays and garnet cover configurations. The arrows show the entry port and propagation direction of the back-reflected light. 
also, we demonstrate that increasing the number of guiding channels leads to better results.

Figure 1 depicts a schematic diagram of the optical isolator structures under investigation in this work. We focus on silicon-on-insulator platforms where nonreciprocity is introduced into the arrays via iron garnet cover layers magnetized transversely to the propagation direction. Transversely magnetized waveguides are known to experience a nonreciprocal phase shift in opposite propagation directions [3-5]. It is assumed that a magneto-optic gyrotropy gradient has been established across the array via composition gradients, either as a result of the selective deposition of different garnet compounds on each of the waveguide elements, or via a lateral combinatorial-materials-synthesis, represented schematically in Fig. 1(c). Moreover, the array is designed to maintain a null gradient in the propagation constant between the adjacent waveguides in the forward direction. This, in turn, gives rise to a finite linear ramp in propagation constant $\delta \beta^{b}$ between the adjacent normal modes in the backward propagation direction [11-16].

As shown in Fig. 1, we examine structures having 2, 3, and 5 waveguides. In all cases, the forward input beam is launched into channel W1 and exits through the rightmost waveguide element (W2, W3, or W5). The distribution of the coupling coefficients is also indicated in the figure. As has been predicted using the CMT, perfect signal transfer in these systems will occur after propagation distances $(1, \sqrt{2}, \sqrt{6}) \pi / 2 \kappa$, for the two-, three-, and five-channel arrays, where $\kappa$ is the coupling constant in the middle of the array [12].

On the other hand, the reflected light in channels W2, W3, or W5 will undergo Bloch oscillations and revival effects will take place after a distance $2 \pi / \delta \beta^{b}$ (a Bloch period). Evidently, there are two independent "knobs" that can be used to tune the performance of this optical isolator device, namely the coupling constant $\kappa$ and the propagation constant step $\delta \beta^{b}$.

While this CMT approach is fast and provides accurate results, it is important to take its limitations into account. We do so by employing a semi-vectorial beam propagation technique to calculate the isolation ratios associated with the TM modes that are propagating in the optical structures shown in Fig. 1. Before outlining our results, we note that two key assumptions are presupposed in our simulations. First, we assume that the gyrotropic, nonreciprocal effect in each channel can be independently controlled by engineering the garnet layer covering this same individual ridge waveguide. Second, all channels have been engineered to produce the same propagation constant throughout the array in the forward direction. As a consequence, they exhibit different propagation constants in the backward direction, due to the gradient in magneto-optic gyrotropy a necessary condition for surface Bloch oscillations [12].

In all simulations, we assume $2-\mu$ m-wide 300 -nm-thick silicon-on-insulator ridges with $20 \mathrm{~nm}$ ridge steps $h$ [see Fig. 1(c)]. The three different arrays we analyze are designed to exhibit the same maximum nonreciprocal phase shift in the input waveguide that is located at one edge of the array (labeled in Fig. 1 as W1), and a null nonreciprocal phase shift in the output channel at the other edge of the array (labeled W5, for five channels, W3, for three channels, or W2, for two channels). We assume a realistic scenario, with the nonreciprocal phase-shift in W1 corresponding to a cerium-substituted iron garnet cover layer with one cerium ion per formula unit [5-8]. Changes in mode profiles corresponding to nonreciprocal effects are taken into account by adjusting the material index of the silicon layer. Reflections at the ends of the waveguides are not accounted for. Values for the nonreciprocal phase-shifts correspond to those for $\mathrm{Ce}_{\mathrm{x}} \mathrm{Y}_{3-\mathrm{x}} \mathrm{Fe}_{5} \mathrm{O}_{12}$ on silicon-on-insulator at different substitution levels [8]. All calculations are done at $1550 \mathrm{~nm}$ wavelength.

The two- and three-channel arrays have the same interwaveguide coupling constant $\kappa$. This is also the same coupling constant between the inner channels of the five-waveguide array. Its outer channels are coupled to the corresponding adjacent ones according to the rules for coherent optical transport, as detailed in [12]. These coupling constants are distributed from one side of the array to the other according to the following pattern: $\sqrt{2 / 3} \kappa, \kappa, \kappa, \sqrt{2 / 3} \kappa$. In all cases, adjusting the ridge waveguide separations controls the coupling constants.

Negligible absorption loss in the silicon core and garnet cover were assumed in our simulations. We estimate the forward losses to be less than one decibel per millimeter (device length) if garnet-cover-induced absorption and scattering losses are included [17].

In all simulations, we assume that the backwardpropagating optical beam is launched into the right most channel through an optical fiber. We consider two different scenarios. In the first scenario, the input beam is first allowed to settle into a superposition of normal modes of the array after the transit coupling to radiation modes. The corresponding array is shown in Fig. 2(a). Under these conditions, it is clear from bars $\mathrm{A}$ in Fig. 3 and column 1 of Table 1 that increasing the number of guiding channels leads to a dramatic improvement in the performance. This result can be explained by noting that the

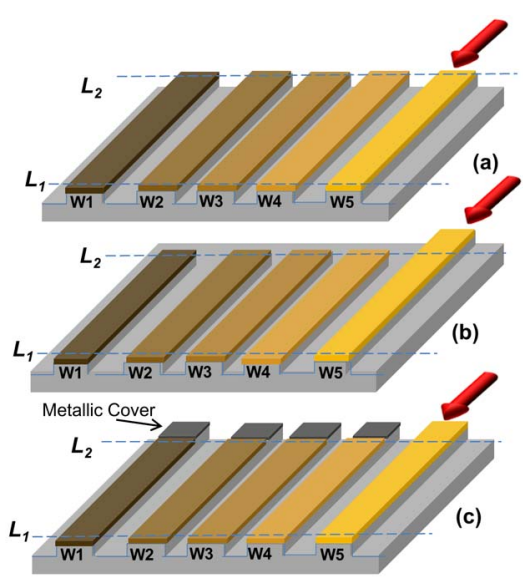

Fig. 2. (a) Backward propagation configuration. The fiber input beam is first allowed to settle into a superposition of normal array modes. (b) The backward propagating beam is first made to converge to a single-waveguide optical mode before coupling into the array. (c) The backward propagating beam is allowed to converge into a stable mode in the backward-launch waveguide. Metallic cover layers before the input plane $L_{2}$ function as absorbers to prevent the radiated light from reaching the other array channels. 


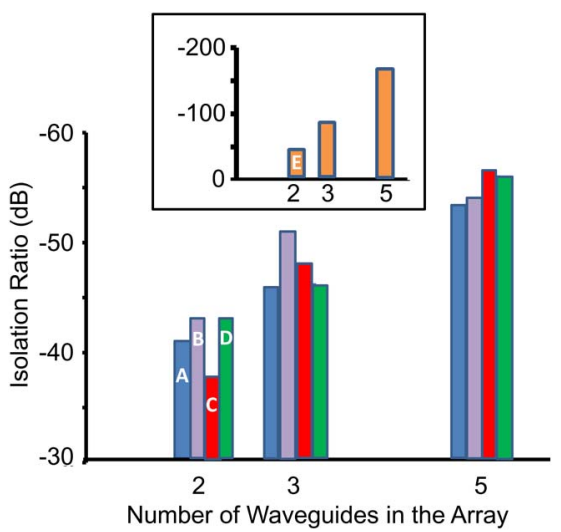

Fig. 3. Isolation ratios versus number of waveguides in the array, calculated in five different ways. All of them show a progression to higher isolation ratios as the number of waveguides increases. (a) Isolation ratio obtained from beam-propagation simulation for the case of a launched fiber mode that has been allowed to convert into a stable linear superposition of array modes (blue); (b) isolation ratio obtained from beam propagation simulation for the case of a single-waveguide mode launch after stabilization in the array at the input (violet); (c) fractional power at launch contained in $\mathrm{W} 1$ relative to the power in the launch waveguide (red); (d) isolation ratios for random departures up to one percent in coupling coefficients from the design values, calculated by the CMT (green). (e) Inset: isolation ratios for random departures up to one percent in propagation constants from design values, calculated by the CMT (orange).

next nearest neighbor coupling is much weaker than nearest neighbor coupling, and thus the evanescent tail coupling between the right most and left most channels at the sharp discontinuity of plane $L_{1}$ becomes negligible as the number of intermediate waveguide elements increases. However, we note that due to energy transfer (mode conversion and radiation losses) from the rightmost channel to the rest of the array at the backward input plane $L_{2}$, the isolation ratio is still lower than that predicted by CMT. Bars C in Fig. 3 and column 3 in Table 1 , show a small but not negligible fractional power (labeled FP) in W1 after the beam stabilization inside the array at the backward input end. FP is defined as the ratio between the power contained in the backward input waveguide and the outermost waveguide channel (W1).

Next we consider a different scenario, depicted in Fig. 2(b), where the backward propagating beam is first made to converge to a single-waveguide optical mode before it evanescently couples to the array. The initial results of our simulations show an unexpected drop in the values of isolation ratios in this case, dropping to $27 \mathrm{~dB}$

Table 1. Isolation Ratios (IR), Fractional Power (FP) for BPM Study (in dB) ${ }^{a}$

\begin{tabular}{lccccc}
\hline $\begin{array}{l}\text { No of } \\
\text { WG } \\
\text { Channels }\end{array}$ & $\begin{array}{c}\text { IR Fiber } \\
\text { Launch } \\
(\mathrm{BPM})\end{array}$ & $\begin{array}{c}\text { IR Single- } \\
\text { Mode } \\
\text { Launch } \\
\text { (BPM) }\end{array}$ & $\begin{array}{c}\text { FP at } \\
\text { Launch } \\
(\mathrm{BPM})\end{array}$ & $\begin{array}{c}\text { IR } \\
\text { Variation } \\
\text { in } \kappa \\
\text { CMT 1\% }\end{array}$ & $\begin{array}{c}\text { IR } \\
\text { Variation } \\
\text { in } \delta \beta^{b} \\
\text { CMT 1\% }\end{array}$ \\
\hline 2 & 41 & 43 & 37 & 43 & 43 \\
3 & 47 & 51 & 48 & 46 & 87 \\
5 & 53 & 54 & 57 & 56 & 167 \\
\hline
\end{tabular}

${ }^{a}$ Perturbed CMT Calculations (in dB). for the three- and five-channel arrays. Upon close inspection, we find that some of the energy leaked from the input beam to the radiation modes during the early stages of propagation before the beam is stabilized is directly coupled to the array. This, in turn, produces undesirable optical intensity at the otherwise isolated channel. In order to overcome this problem, we propose using radiation absorbers, shown in Fig. 2(c), to eliminate this freespace coupling. These absorbers are assumed to be made of silicon with metallic covers and are easy to fabricate on the same chip. Semi-vectorial beam propagation analysis indeed confirms a remarkable increase in the isolation ratios when absorbers are added in. This is clearly indicated in bars B of Fig. 3 and column 2 of Table 1 (the isolation ratios are each labeled as IR). Our simulations also demonstrate that, similarly to the previous case, the performance of these arrangements scales up with the array size. We estimate a negligible increase in forward loss $(0.03 \mathrm{~dB})$ due to these absorbers.

These results suggest a consistent pattern: using higherchannel multiplicity arrays can largely offset the performance degradation due to the effects of radiation and evanescent tail coupling. Along these lines, it is interesting to note that coupled-mode analysis demonstrates the same trend. This can be illustrated by examining the performance of the optical waveguide isolators when the system's parameters deviate from their ideal values. Columns 4 and 5 of Table 1 and bars D and $\mathrm{E}$ in Fig. 3 present these data when the coupling coefficients and the propagation constants are randomly varied within $1 \%$ of their design values in one direction while fixing the device length. This corresponds to $6 \mathrm{~nm}$ departures from the design values in inter-waveguide separations and more than $1 \mu \mathrm{m}$ deviations in waveguide widths. Systematic 10\% deviations in coupling constants, corresponding to $46 \mathrm{~nm}$ departures from the design in inter-waveguide separations yield isolation ratios of 19.9, 39, and $52 \mathrm{~dB}$ for two-, three-, and fivewaveguide arrays, respectively. The isolation ratios are normalized to the performance of a two-waveguide array computed at design values by the beam propagation method (BPM) for comparison. Evidently, this CMT analysis confirms our conclusion that the device performance improves drastically as the number of waveguide channels increases. This can be understood by noting that the impact of these random variations on the isolation ratios will tend to average out with the expansion of the design parameters' space.

In summary, we have analyzed the effect of mode conversion and fabrication tolerances on the optical performance of the novel type of isolator proposed in $[11,12]$. We find that the figure of merit increases in magnitude with the number of channels in the array; that is, it depends on the channel multiplicity of the array. A mechanism to dial up isolation performance and robustness in nonreciprocal coupled-waveguide arrays is thus proposed and analyzed. The functional mechanism also allows for the independent control of forward transmission and enables low forward loss based on coherent optical transport.

The authors M. L. and T. C. acknowledge support from the Air Force Research Laboratory (AFRL/RY) and the Air Force Office of Scientific Research (Dr. Arje 
Nachman), through the Advanced Materials, Manufacturing, and Testing Information Analysis Center (AMMTIAC) Contract FA4600-06-D-0003 DO\#0048.

\section{References}

1. T. Shintaku, Appl. Phys. Lett. 66, 2789 (1995).

2. N. Bahlmann, M. Lohmeyer, O. Zhuromsky, H. Dötsch, and P. Hertel, Opt. Commun. 161, 330 (1999).

3. J. Fujita, M. Levy, R. M. Osgood, Jr., L. Wilkens, and H. Dötsch, Appl. Phys. Lett. 76, 2158 (2000).

4. R. L. Espinola, T. Izuhara, M.-C. Tsai, R. M. Osgood, Jr., and H. Dotsch, Opt. Lett. 29, 941 (2004).

5. Y. Shoji, T. Mizumoto, H. Yokoi, I.-W. Hsieh, and R. M. Osgood, Jr., Appl. Phys. Lett. 92, 071117 (2008).

6. L. Bi, J. Hu, P. Jiang, D. H. Kim, G. F. Dionne, L. C. Kimerling, and C. A. Ross, Nat. Photonics 5, 758 (2011).

7. M.-C. Tien, T. Mizumoto, P. Pintus, H. Kromer, and J. E. Bowers, Opt. Express 19, 11740 (2011).
8. T. Mizumoto, R. Takei, and Y. Shoji, IEEE J. Quantum Electron. 48, 252 (2012).

9. M. Levy, H. Hegde, F. J. Cadieu, R. Wolfe, V. J. Fratello, and R. M. Osgood, Jr., IEEE Photon. Technol. Lett. 8, 903 (1996).

10. A. Alberucci and G. Assanto, Opt. Lett. 33, 1641 (2008).

11. R. El-Ganainy and M. Levy, IEEE Photon. J. 6, 1 (2014).

12. R. El-Ganainy, A. Eisfeld, M. Levy, and D. N. Christodoulides, Appl. Phys. Lett. 103, 161105 (2013).

13. M. Levy and P. Kumar, Opt. Lett. 35, 3147 (2010).

14. P. Kumar and M. Levy, Opt. Lett. 36, 4359 (2011).

15. P. Kumar and M. Levy, Opt. Lett. 37, 3762 (2012).

16. R. El-Ganainy, P. Kumar, and M. Levy, Opt. Lett. 38, 61 (2013).

17. S.-Y. Sung, A. Sharma, A. Block, K. Keuhn, and B. J. H. Stadler, J. Appl. Phys. 109, 07B738 (2011). 